\title{
ESTUDO DE ALCOOLISMO E TABAGISMO ASSOCIADOS COM A TUBERCULOSE PULMONAR
}

* Márcia Caron Ruffino

\begin{tabular}{l|l|}
$/ 05$ \\
\hline
\end{tabular}

RUFFINO, M.C. - Estudo de alcoolismo e tabagismo associados com a tuberculose pulmonar. Rev. Bras. Enf.; DF, 32 : 48-60, 1979.

\section{INTRODUÇĀO}

A tuberculose continua sendo um sério problema de Saúde Pública em muitos países, sendo que, a Organização Mundial de Saúde (WHO, 1964) assinalava que a tuberculose persistia como problema da maior importância em quase todos os paises.

Vários autores têm evidenciado alguns elementos como constituindo fatores risco para a tuberculose.

FELDMAN (1961), assinala que o alcoolismo é um fator que altera negativamente a resistência individual.

$\mathrm{Na}$ literatura encontramos alguns autores que apontam o alcoolismo como possivel fator risco associado à tuberculose (OLIN, 1966; OLIN, 1966 a; ROBBINS, 1969; BANNER, 1973).

Quanto ao tabagismo, vários autores têm assinalado sua importância com relação à tuberculose (LOWE, 1956; EDWARDS, 1957).
Em vista do exposto, pretendemos no presente trabalho estudar o alcoolismo e tabagismo como possiveis fatores risco da tuberculose pulmonar em nosso meio.

\section{OBJETIVOS}

Avaliar a associação entre o alcoolismo e hábito de fumar com a tuberculose pulmonar.

\section{MATERIAL E MÉTODOS}

A metodologia utilizada foi a de caso-controle, segundo modelo apregoado por MacMAHON \& PUGH (1970); FORATTINI (1976).

\subsection{Casos}

Definição: Foi chamado de caso de tuberculose pulmonar, os pacientes internados em hospitais especializados

\footnotetext{
- Docente da Escola de Enfermagem de Ribeirão Preto, USP. Departamento de Enfermagem Geral e Especializada.

Trabalho apresentado no XXX CBEn - Belém - 1978.
} 
RUFFINO, M.C. - Estudo de alcoolismo e tabagismo associados com a tuberculose puimonar. Rev. Bras. Enf.; DF, $32: 48-60,1979$.

(dentro do Estado de São Paulo), em tratamento com tuberculostáticos.

Fonte: Portadores de tuberculose pulmonar, internados no Sanatório Nestor Goulart dos Reis, em Américo Bra.siliense - Araraquara, Estado de São Paulo, e pacientes internados no Abrigo Ana Diedericksen, Ribeirāo Preto, Estado de São Paulo.

Critério de Seleção: Pacientes de ambos os sexos, qualquer raça ou cor, do grupo etário de 10 a 80 anos, que tivessem condiçōes de lucidez para responder a entrevista.

Tamanho amostral: Foram entrevistados um total de 427 pacientes.

\subsection{Controles}

Definição: Pacientes internados em um serviço de assistência médica, com outras patologias, que nāo fosse a tuberculose.

Fonte: Pacientes internados no Hospital das Clínicas de Ribeirão Preto.

Tamanho da amostra: Foram entrevistados 427 controles, ou seja, o número correspondente ao número de casos.

\subsection{Variáveis que foram pareadas}

Para cada caso, tomou-se um controle, pareado com respeito às seguintes variáveis: idade, sexo, estado civil, procedência.

3.4 Alcoolismo e tabagismo obser.vados nos grupos casos e controles

Tabagismo: Quanto ao hábito de fumar, no momento da entrevista os pacientes eram classificados em fumantes e não fumantes. Aos fumantes foi perguntado qual o tempo que apresen.tou o hábito de fumar precedendo à doença (em meses); qual a média de cigarros fumados por dia no último ano precedendo à doença, média essa que foi classificada em $0,1-4 ; 5-14 ; 15-24$ cigarros por dia. Foi investigado também qual o tipo de cigarro utilizado, classificado em: papel, palha, cachimbo, outro.

Ingestão alcoólica: Foi perguntado qual o hábito de ingestão de álcool no periodo que precedeu à doença (periodo menor de 2 anos e período de 2 a 4 anos-.

Os entrevistados foram divididos em abstêmios, bebedores moderados, bebedores excessivos e bebedores adictos (classificação essa adaptada daquela apregoada por MARCONI, 1959).

Quanto ao tipo de bebida predominantemente ingerida, foi classificado baseado nas bebidas passiveis de serem consumidas em nossa área, ou seja: aguardente, cerveja, vinho e outros.

Quanto ao ritmo de ingestāo, foi classificada como: mensal ou ocasional, semanal, diário.

Foi investigado também, qual o hábito de ingestão precedendo o diagnóstico por um periodo de 2 a 4 anos.

3.5 Outras variáveis observadas nos grupos casos e controles

Profissão
Religião
Cor
Instruçāo
Categoria de internação
Grau de evolução da doença - Casos
Diagnóstico - Controles.

\subsection{Instrumento de medida}

Foi realizada através de uma entrevista pessoal com o paciente, dirigida no sentido de preencher um questionário pré-estabelecido. 
RUFFINO, M.C. - Estudo de alcoolismo e tabagismo associados com a tuberculose pulmonar. Rev. Bras. Enf.; DF, 32 : 48-60, 1979.

Para entrevistar os casos, bem como os controles, foi utilizado um protocolo de investigação (Anexo 1).

As entrevistas (com o caso estudo e com o seu controle), foram feitas todas pela mesma pessoa.

Os quesitos do questionário versaram especificamente sobre identificaçāo pessoal do caso, com as variáveis que foram pareadas $e$ aquelas que foram medidas.

\subsection{Método Estatístico Foi usado o teste $\mathrm{X}^{2}$.}

\section{RESULTADOS E DISCUSSĀO}

Na tabela 1 é apresentada a distribuiçāo dos casos e controles, segundo o sexo e grupo etário. Foram entrevistadas 854 pessoas, sendo 214 do sexo masculino (casos e/ou controles) e 213 do sexo feminino (casos e/ou controles).

$\mathrm{Na}$ tabela 2 é apresentada a distribuição dos casos e dos controles, segundo estado civil e procedência.

O tempo que apresentou hábito de fumar precedendo à doença, assim como a média de cigarros fumados por dia (no último ano que precedeu o diagnóstico da doença) e o tipo e/ou forma de tabaco predominantemente utilizado pelos casos e controles, são apresenta- dos respectivamente nas tabelas 3,4 e 5 .

Na tabela 6 é apresentada a distribuição dos casos e controles, segundo o hábito da ingestão alcoólica no periodo menor que 2 anos precedendo ao diagnóstico da doença; a distribuição segundo a mesma variável, para o período compreendido entre 2 a 4 anos precedendo ao diagnóstico é visto na tabela 7 .

A distribuição dos casos e/ou controles (habituados à ingestāo alcoólica). segundo o ritmo e tipo de bebida predominantemente consumida, nos últimas 2 anos precedendo ao diagnóstico, e no periodo de 2 a 4 anos precedendo ao diagnóstico, são apresentadas respectivamente nas tabelas 8 e 9 .

O grau de classificaçāo dos casos de tuberculose internados (segundo a $\mathrm{Na}$ tional Tuberculosis Association - NTA) é visto na tabela 10 .

\section{Tabagismo:}

A partir da tabela 3, verifica-se que a porcentagem de não fumantes é maior nos controles $(53,86 \%)$ que entre os casos $(\mathbf{4 5 , 4 3 \% )}$.

Reconstruindo com os dados da tabela 7 , uma tabela $2 \times 2$, como a tabela abaixo, teremos:

\begin{tabular}{lccc}
$\begin{array}{l}\text { Apresenta } \\
\text { hábito de } \\
\text { fumar }\end{array}$ & Casos & Controles & Total \\
\hline SIM & 233 & 197 & 430 \\
\hline NAO & 194 & 230 & 424 \\
\hline TOTAL & 427 & 427 & 854 \\
\hline
\end{tabular}

que resultou ao teste de associação um $\mathrm{X}^{2}=6,07(\mathrm{p}<0,05)$, isto é, existe uma associação entre hábito de fumar e a doença (estatisticamente significante com um co de $5 \%$ ).
Ainda na tabela 3 , na distribuiçāo pode-se observar também que entre os fumantes, os casos parecem apresentar o hábito de fumar há mais tempo que os controles, embora o teste não eviden- 
RUFFINO, M.C. - Estudo de alcoolismo e tabagismo associados com a tuberculose pulmonar. Rev. Bras. Enf.; DF, $32: 48-60,1979$.

ciasse diferença estatisticamente significante $\left(\mathrm{X}^{2}=11,92 ; 0,10<\mathrm{p}<0,20\right)$.

Os dados da tabela 4 como um todo, não evidenciam diferença estatisticamente significante $\left(\mathrm{X}^{2}=7,00\right.$; $0,05<\mathrm{p}<0,10$ ), ou seja, não existe diferença entre casos e controles quanto ao número de cigarros fumados por dia (entre os fumantes).

Da mesma forma, não existiu diferença significante quanto ao tipo de tabaco consumido entre casos e controles (tabela 5 ) $\left(\mathrm{X}^{2}=3,55 ; 0,10<\mathrm{p}<0,20\right)$.

Concluímos portantö, que parece haver associação entre hábito de fuma: e tuberculose (enquanto classificados como fumantes e não fumantes).
Se tomarmos apenas os fumantes, não existe contudo diferença quanto ao tempo que apresenta hábito de fumar e/ou quanto ao tipo de tabaco consumido entre os casos e os controles.

\section{Alcoolismo:}

Na tabela 6, verifica-se que a porcentagem de abstêmios é maior nos controles $(70,96 \%)$ que entre os casos $(55,73 \%)$, dados estes referentes ao hábito de ingestão alcoólica no periodo de 2 anos ou menos precedendo o diagnóstico.

Reconstruindo com os dados da tabela 10, uma tabela $2 \times 2$, teremos:

Hábito de

ingestão alcoólica

Casos Controles Total

\begin{tabular}{cccc}
\hline SIM & 189 & 124 & 313 \\
NAO & 238 & 303 & 541 \\
\hline TOTAL & 427 & 427 & 854 \\
\hline
\end{tabular}

que resultou ao teste de associação um $\mathrm{X}^{2}=21,30(\mathrm{p}<0,01)$, isto é, existe umi associação entre o hábito de beber e a tuberculose pulmonar (estatisticamente significante).

Analisando a tabela 6 como um todo, obtemos um $\mathrm{X}^{2}=41,65(\mathrm{p}<0,01)$ sendo que existe diferença significante, sendo que esta diferença parece ser mais evidente para os bebedores excessivos.

Se retirarmos da tabela 6, o grupo dos abstêmios, e analisarmos apenas os bebedores, teremos:

Hábito de

ingestão alcoólica

Casos Controles $\quad$ Total

\begin{tabular}{lccc}
\hline Moderado & 126 & 111 & 237 \\
Excessivo & 48 & 10 & 58 \\
Adicto & 15 & 3 & 18 \\
\hline TOTAL & 189 & 124 & 313 \\
\hline
\end{tabular}


RUFFINO, M.C. - Estudo de alcoolismo e tabagismo associados com a tuberculose pulmonar. Rev. Bras. Enf.; DF, $32: 48-60,1979$.

que resulta ao teste de associação um $\mathrm{X}^{2}=21,26(\mathrm{p}<0,01)$ sendo que novamente a diferença é mais evidente no grupo dos bebedores excessivos.

$\mathrm{Na}$ tabela 7 , verifica-se que a porcentagem de abstêrnios é maior nos controles $(\mathbf{7 0 , 2 5 \% )}$ que entre os casos $(57,37 \%)$, dados estes referentes ao hábltơ de ingestão alcoólica, no período de 2 a 4 anos precedendo o diagnóstico.
Analisando-se a tabela como um todo, o teste de associação nos dá evidências de que existe associação significante, pois $\mathrm{X}^{2}=27,57(\mathrm{p}<0,01)$. De novo, a evidência é maior para o grupo dos bebedores excessivos.

Reconstruindo a tabela 7, de forma a permitir a análise dos grupos de bebedores (eliminando os abstêmios) temos:

\section{Hábito de}

ingestão alcoólica

Casos Controles Total

\begin{tabular}{lrrr}
\hline Moderado & 118 & 104 & 222 \\
Excessivo & 49 & 21 & 70 \\
Adicto & 15 & 2 & 17 \\
\hline TOTAL & 182 & 127 & 309 \\
\hline
\end{tabular}

Isto nos dá um $\mathrm{X}^{2}=12,64(\mathrm{p}<0,01)$ havendo portanto associação significante entre diferentes hábitos de ingestão alcoólica (por 2 a 4 anos antes do diagnóstico) e a tuberculose pulmonar. Ocorre contudo que a maior diferença encontra-se no grupo dos adictos.

Dado que são não abstêmios (isto é, que são bebedores) não há diferença significante quanto ao tipo de bebida consumida e/ou ritmo de ingestão entre os casos e controles (tabelas 8 e 9).

Concluímos assim, que há associação entre alcoolismo e tuberculose pulmonar. Entre os casos predomina os alcoólatras excessivos menor que 2 anos precedendo ao diagnóstico; para o período de 2 a 4 anos precedendo o diag.nóstico predominam os alcoólatras adic.. tos entre os casos.

\section{CONCLUSÓES}

1. Há associação entre tabagismo e tuberculose pulmonar.

1.1 Entre os fumantes, não há diferença entre casos e controles quanto ao tempo que apresenta hábito de tabagismo.

1.2 Entre os fumantes, não há diferença entre casos e controles quanto ao tipo de tabaco consumido.

2. Há associação entre alcoolismo e tuberculose pulmonar.

2.1 No periodo menor que 2 anos que precede à doença, é mais forte a associação entre tuberculose pulmonar e bebedores excessivos.

2.2 No periodo de 2 a 4 anos que precede à doença, é mais forte a associação entre tuberculose pulmonar e bebedores adictos.

2.3 Entre os alcoólatras, não há diferença significante quanto ao tipo de bebida consumida e/ou ritmo de ingestão entre os casos e controles.

\section{REFERENCIAS BIBLIOGRÁFICAS}

BANNER, A. S. - Pulmonary function in chromic alcoholism. Amer. Rev. Resp. Dis., 108 (4) :851-857, 1973.

EDWARDS, J. H. - Contribution of cigarette smoking to respiratory disease 
RUFFINO, M.C. - Estudo de alcoolismo e tabagismo associados com a tuberculose pulmonar. Rev. Bras. Enf.; DF, $32: 48-60,1979$.

Brit. J. of Prev. \& Social Medicine, 11 (1) :10-21, 1957.

FELDMAN, J. - Resistência e susceptibilidade na tuberculose. In: Curso de $\mathrm{Ti}-$ siologia. Belo Herizonte, Departamento de Publicaçōes do Diretório Acadêmico Alfredo Balena, 1961, p. 93-106.

FORATTINI, O. P. - Estudo de casoscontroles, in: Epidemiologia Geral. S. Paulo, Edgard Blïrrer/EDUSP, 1976, p. 117-131.

HADDAD, N. - Inquérito epidemiológico sobre cardiopatias crônicas em um bairro de Ribeirão Preto - São Paulo, Brasil. Tese de Docência-Livre na Cadeira de Higiene e Medicina Preventiva e Estatística, Ribeirão Preto, São Paulo, Brasil, 1965.

LOWE, $C$. $R$ - An association between smoking and respiratory tuberculosis. Brit. Med. J. 2:1081-1086, 1956.

MacMAHON, B. \& PUGH, T. F. - Casecontrol studies. In: Epidemiology: prin- ciples and methods. Boston, Little, Brown and Company, 1970, p. 241-282.

MARCONI, J. T. - The concept of alcoholism. Quart. J. Stud. Alcohol 20 (2): 216-235, 1959.

OLIN, J. S. - "Skid Row" Syndrome: a medical profile of the chronic drunkenness offender. Canad. Med. Ass J. 95:205-214, 1966.

OLIN, J. S. \& CRZBOWSKI. S. - Tuberculosis and alcoholism. Canad. Med. Ass. J. 94:999-1001, 1966.

ROBBINS, S. L. - Sistema gastrointestinal. In: Patologia. 3. a edição, Guanabara Koogan. Rio de Janeiro, 1969, p. 815893.

WORLD HEALTH ORGANIZATION - EXPERT COMMTTTEE ON TUBERCULOSIS EIGHTH REPORT - World Health Organization - Technical Roport Series n. ${ }^{\circ}$ 290, Geneva, 1964. 
RUFFINO, M.C. - Estudo de alcoolismo e tabagismo associados com a tuberculose pulmonar. Rev. B ras. Enf.; DF, 32 : 48-60, 1979.

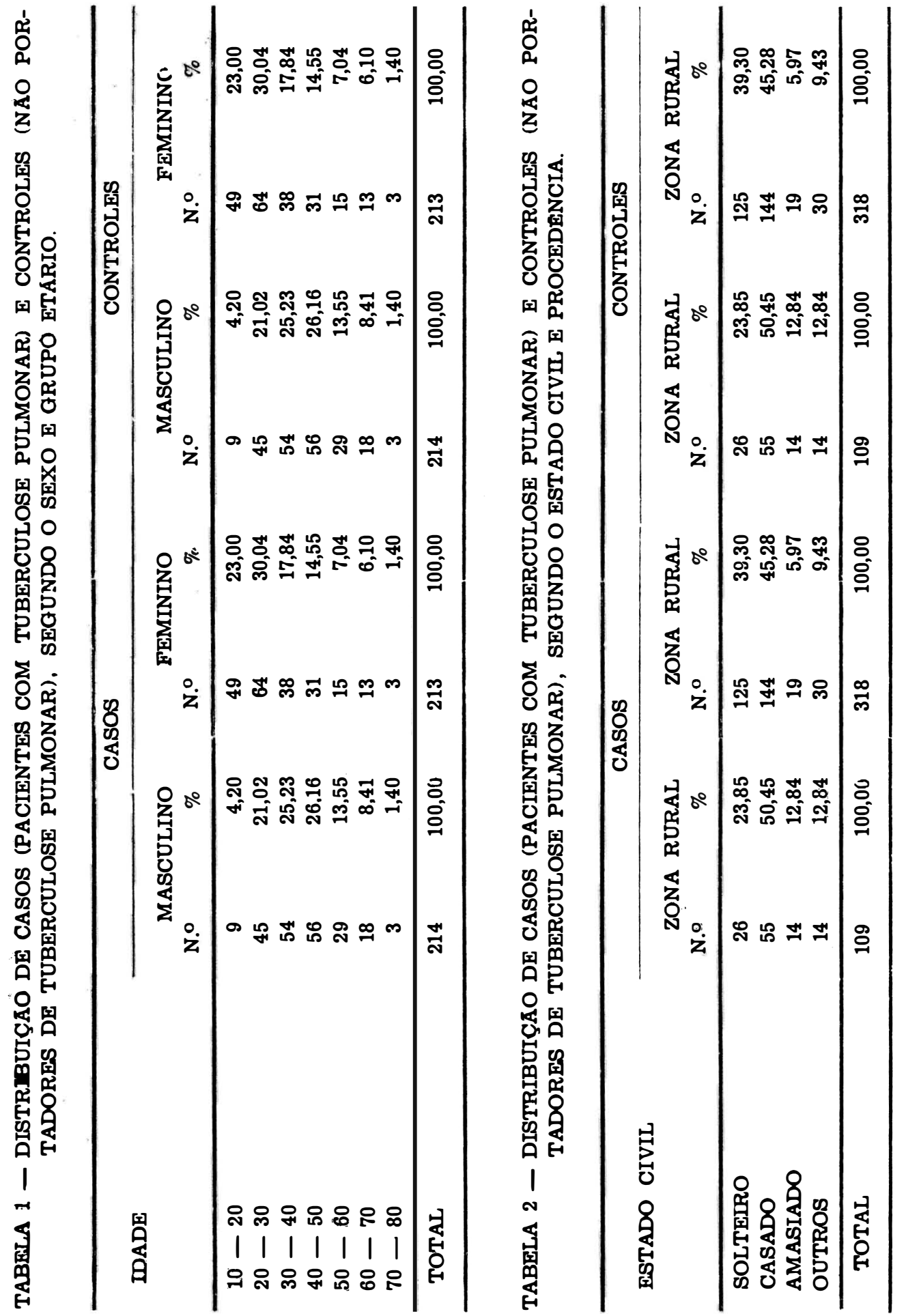


RUFFINO, M.C. - Estudo de alcoolismo e tabagismo associados com a tuberculose pulmonar. Rev. Bras. Enf.; DF, 32 : 48-60, 1979.

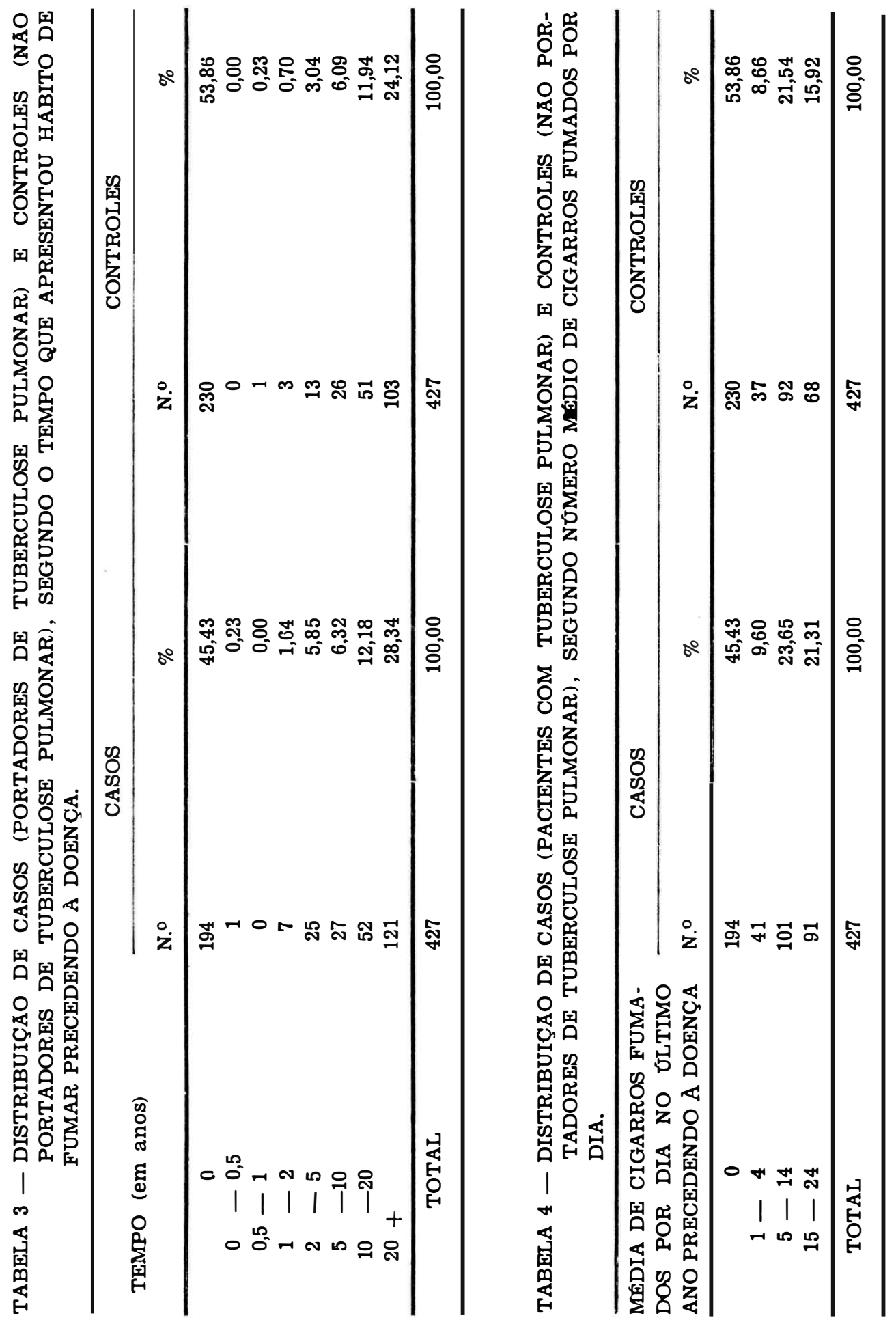


RUFFINO, M.C. - Estudo de alcoolismo e tabagismo associados com a tuberculose pulmonar. Rev. Bras. Enf.; DF, 32 : 48-60, 1979.

TABELA 5 - DISTRIBUIÇAO DE CASOS (PORTADORES DE TUBERCULOSE PULMONAR) E CONTROLES (NAO PORTADORES DE TUBERCULOSE PULMONAR), SEGUNDO O TIPO E/OU FORMA DE TABACO CONSUMIDO.

\begin{tabular}{lrrrr}
\hline TIPO E/OU FORMA DE & \multicolumn{2}{c}{ CASOS } & \multicolumn{2}{c}{ CONTROLES } \\
\cline { 2 - 5 } TABACO CONSUMIDO & N. & $\%$ & N. & $\%$ \\
\hline CIGARRO DE PAPEL & 178 & 76,39 & 149 & 75,63 \\
CIGARRO DE PALHA & 45 & 19,31 & 45 & 22,84 \\
CACHIMBO & 10 & 4,29 & 3 & 1,52 \\
\hline TOTAL & 233 & 100,00 & 197 & 100,00 \\
\hline
\end{tabular}

TABELA 6 - DISTRIBUIÇAO DE CASOS (PACIENTES COM TUBERCULOSE PULMONAR) E CONTROLES (NAO PORTADORES DE TUBERCULOSE PULMONAR), SEGUNDO O HABITO DE INGESTAO ALCOÓLICA, NO PERTODO MENOR QUE DOIS ANOS PRECEDENDO AO DIAGNÓSTICO.

\begin{tabular}{lrrrr}
\hline \multirow{2}{*}{$\begin{array}{c}\text { HABITO DE INGESTAO } \\
\text { ALCOLICA }\end{array}$} & \multicolumn{2}{c}{ CASOS } & \multicolumn{2}{c}{ CONTROLES } \\
\cline { 2 - 5 } & N.o & $\%$ & N.o & $\%$ \\
\hline ABSTEMIOS & 238 & 55,73 & 303 & 70,96 \\
BEBEDOR MODERADO & 126 & 29,50 & 111 & 25,99 \\
BEBEDOR EXECESSIVO & 48 & 11,24 & 10 & 2,34 \\
BEBEDOR ADICTO & 15 & 3,51 & 3 & 0,70 \\
\hline \multicolumn{1}{c}{ TOTAL } & 427 & 100,00 & 427 & 100,00 \\
\hline
\end{tabular}

TABELA 7 - DISTRIBUIÇAO DE CASOS (PORTADORES DE TUBERCULOSE PULMONAR) E CONTROLES (NAO PORTADORES DE TUBERCULOSE PULMONAR) SEGUNDO O HABITO DE INGESTAO ALCOÓLICA, NO PERfODO DE DOIS A QUATRO ANOS PRECEDENDO AO DIAGNÓSTICO.

\begin{tabular}{lrrrr}
\hline \multirow{2}{*}{$\begin{array}{c}\text { HABITO DE INGESTAO } \\
\text { ALCOOLICA }\end{array}$} & \multicolumn{2}{c}{ CASOS } & \multicolumn{2}{c}{ CONTROLES } \\
\cline { 2 - 5 } & N. & $\%$ & N. & $\%$ \\
\hline ABSTEMIOS & 245 & 57,37 & 300 & 70,25 \\
BEBEDOR MODERADO & 118 & 27,63 & 104 & 24,35 \\
BEBEDOR EXCESSIVO & 49 & 11,47 & 21 & 4.31 \\
BEBEDOR ADICTO & 15 & 3,51 & 2 & $0, \therefore 5$ \\
\hline \multicolumn{1}{c}{ TOTAL } & 427 & 109,10 & 427 & $1,0,00$ \\
\hline
\end{tabular}




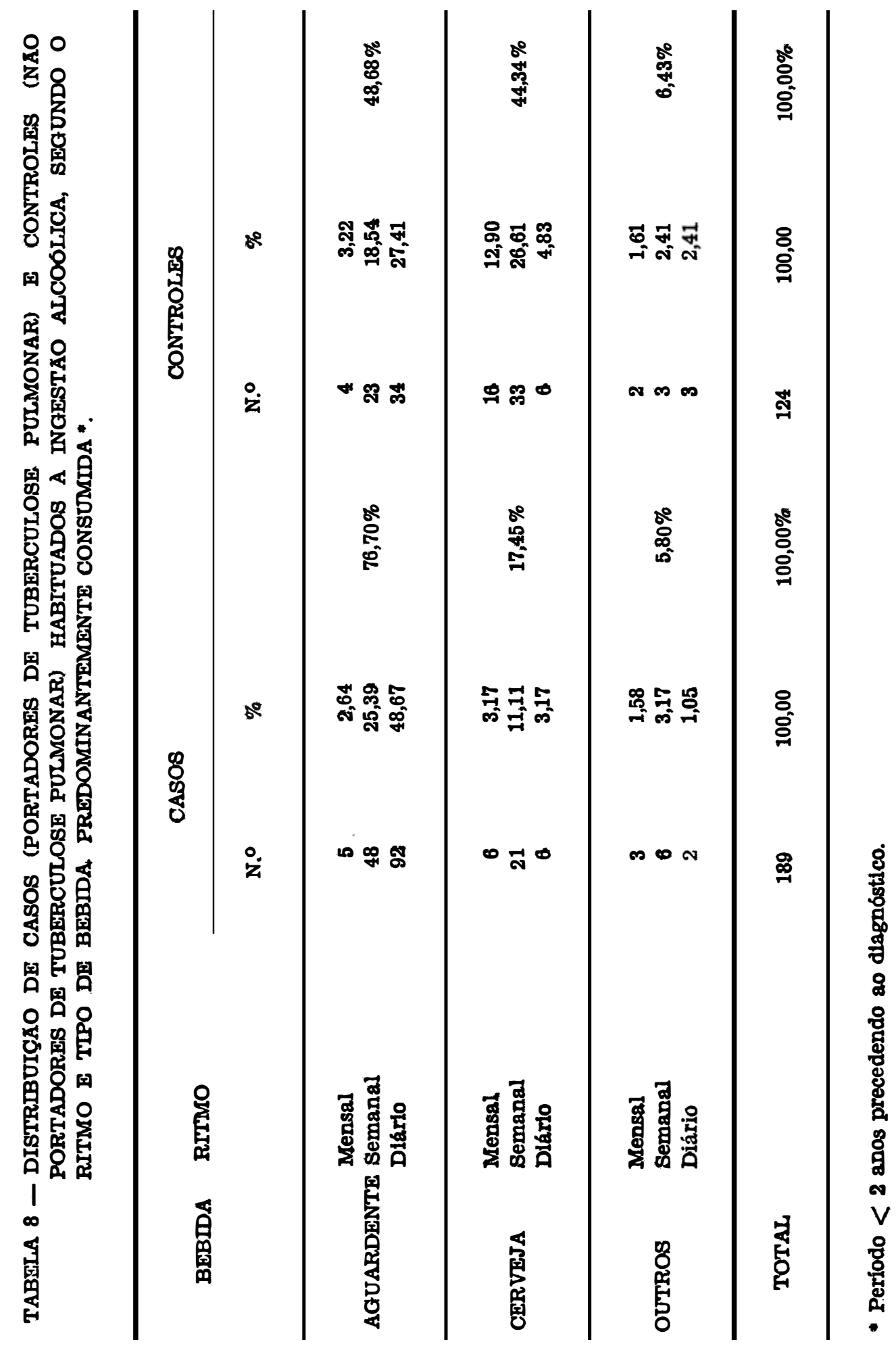


RUFFINO, M.C. - Estudo de alcoolismo e tabagismo associados com a tuberculose pulmonar. Rev. Bras. Enf. DF, $32: 48-60,1979$.

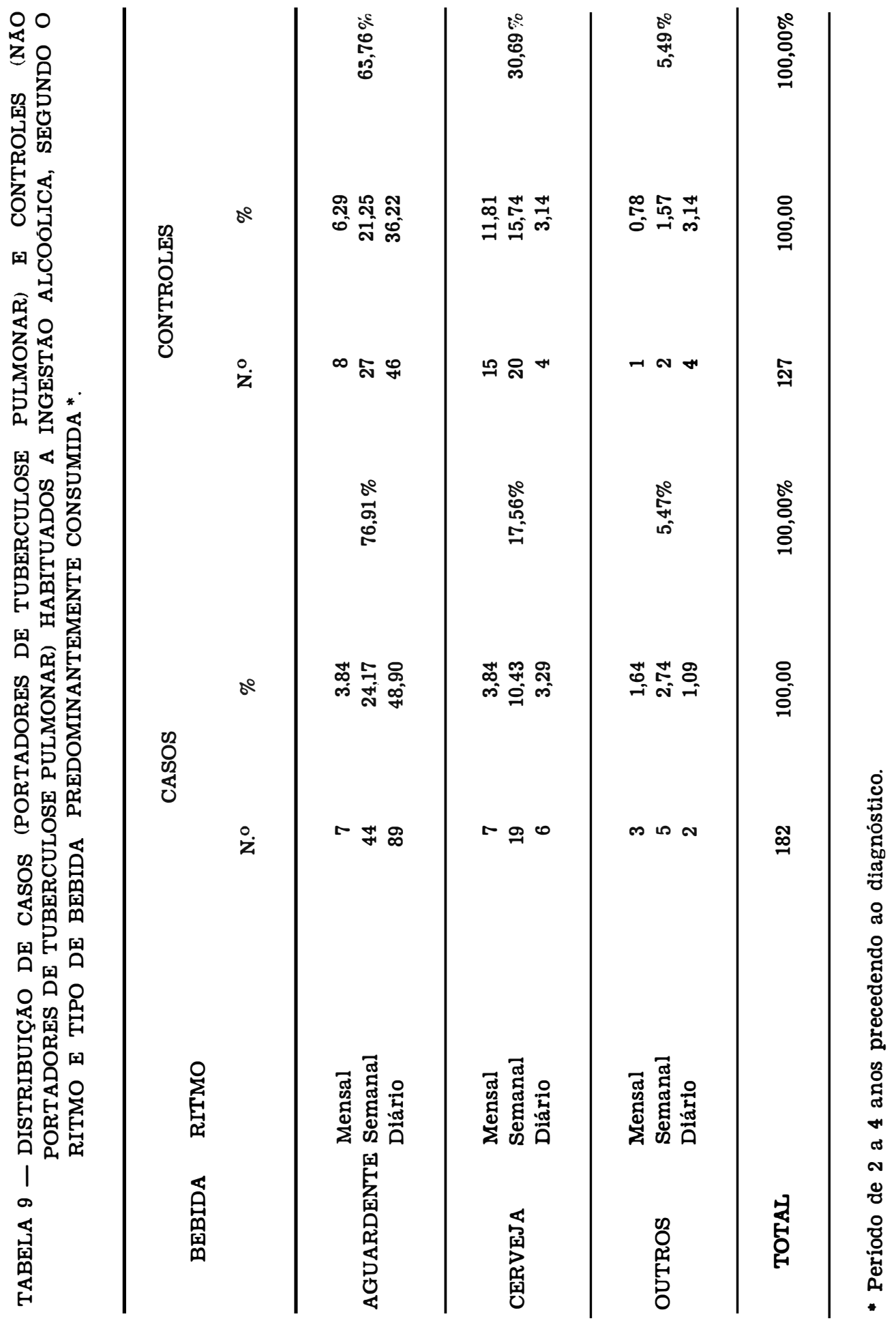


RUFFINO, M.C. - Estudo de alcoolismo e tabagismo associados com a tuberculose pulmonar. Rev. Bras. Enf.; DF, $32: 48-60,1979$.

TABELA 10 -- DISTRIBUIÇÃO DOS CASOS (PACIENTES PORTADORES DE TUBERCULOSE PULMONAR) SEGUNDO O GRAU DE CLASSIFICAÇAO DA DOENÇA.

\begin{tabular}{ccc} 
GRAU DE CLASSIFICAÇAO * & N. ${ }^{*}$ & $\%$ \\
I & 16 & 3,74 \\
II & 99 & 23,18 \\
III & 299 & 70,02 \\
NAO ESPECIFICADO & 13 & 3,04 \\
\hline TOTAI & 427 & 100,00 \\
\hline
\end{tabular}

* Classificação NTA (National Tuberculosis Association). 
RUFFrNO, M.C. - Estudo de alcoolismo e tabagismo associados com a tuberculose pulmonar. Rev. Bras. Enf. DF, 32 : 48-60, 1979.
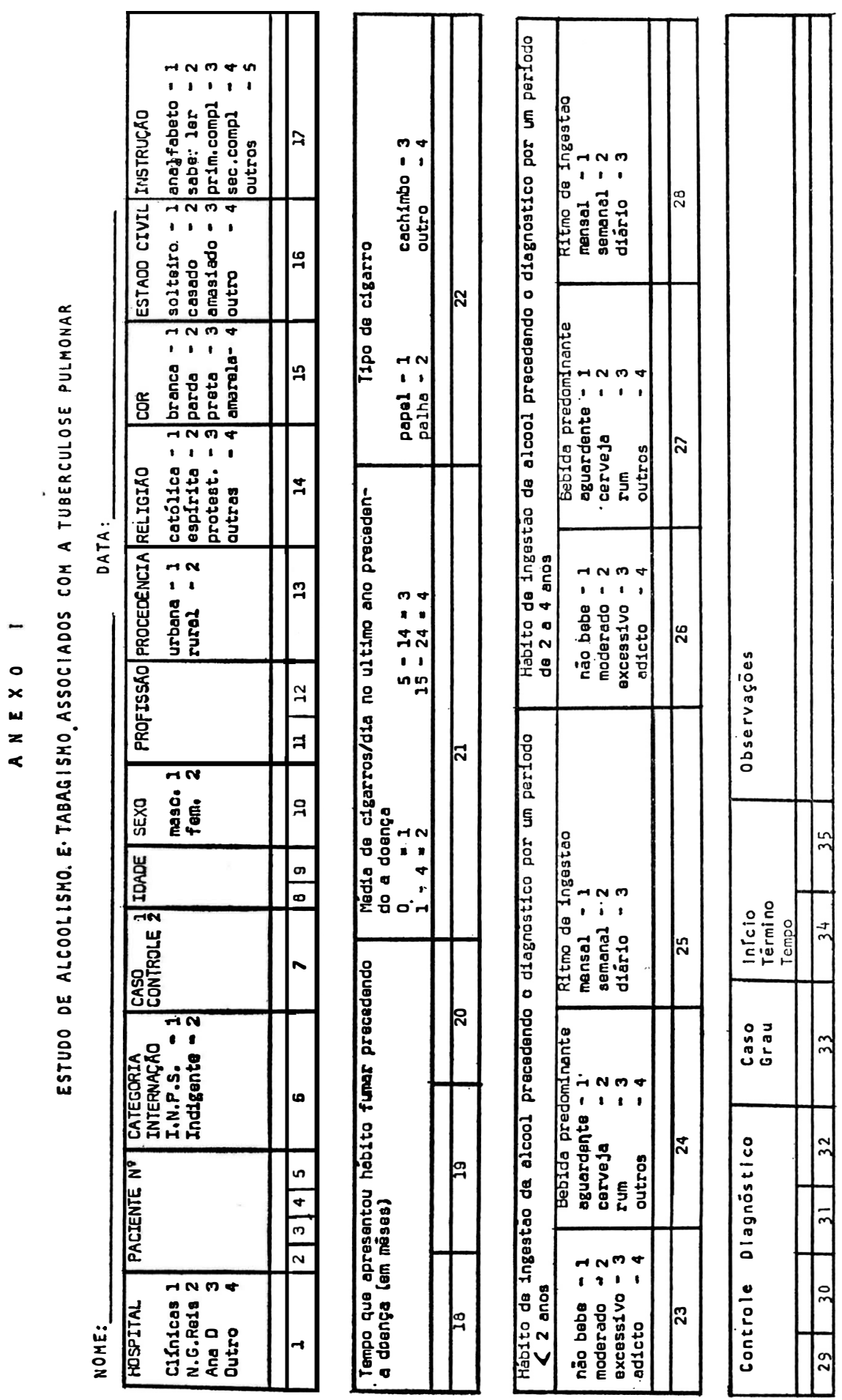